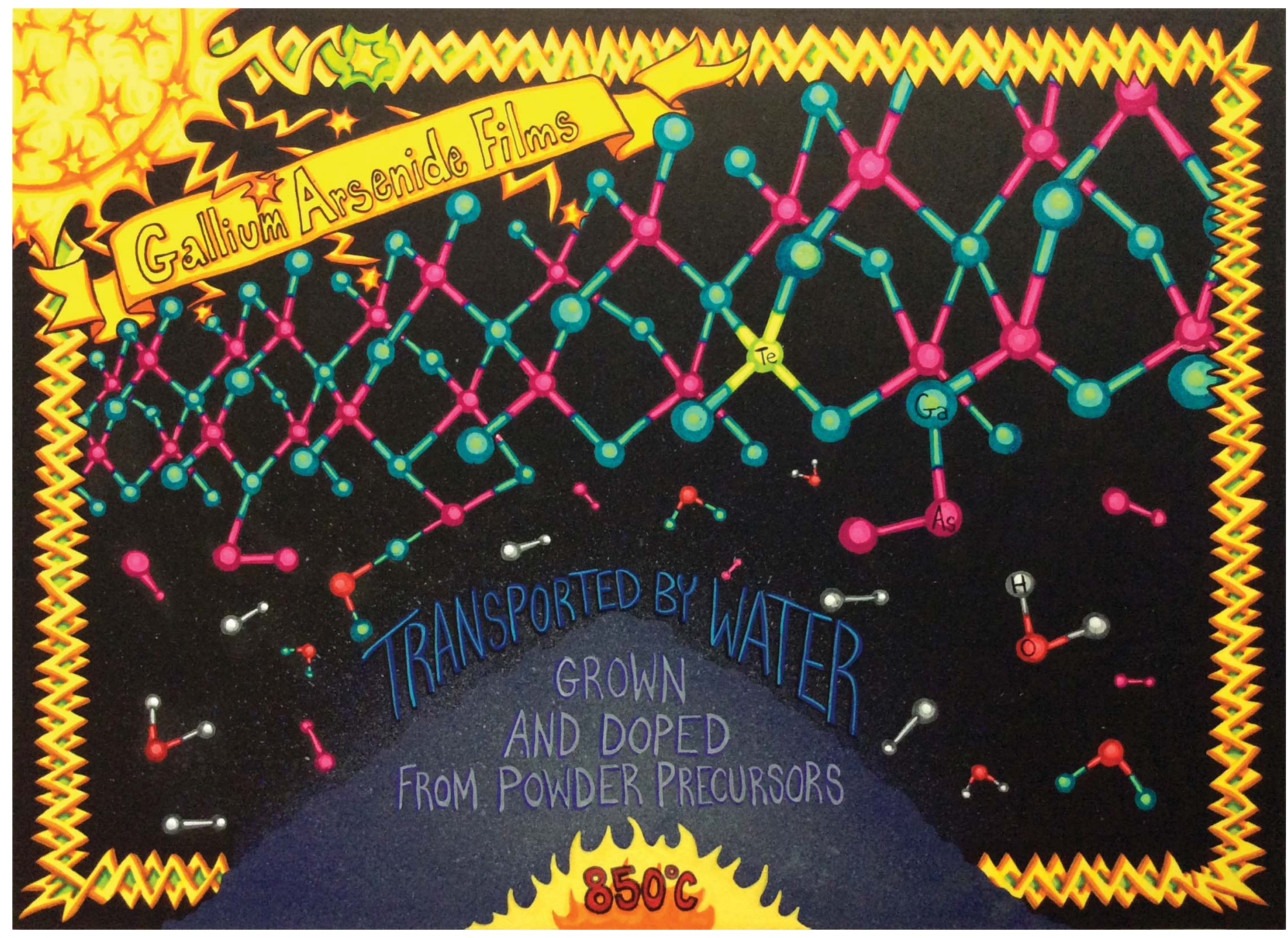

Showcasing research from the laboratories of Dr Shannon Boettcher at University of Oregon, Eugene, USA.

Title: Doping and electronic properties of GaAs grown by close-spaced vapor transport from powder sources for scalable III-V photovoltaics

GaAs and related III-V semiconductors are used in the highestefficiency photovoltaics, but the technology is too expensive for terrestrial application. We report the use of a simple, atmospheric-pressure, vapor-transport technique for the growth of high-quality epitaxial GaAs films with controlled doping using inexpensive GaAs powders as precursors.

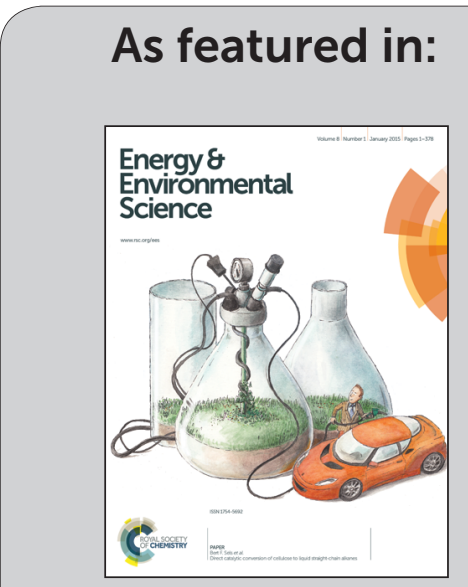

See Shannon W. Boettcher et al., Energy Environ. Sci., 2015, 8, 278.

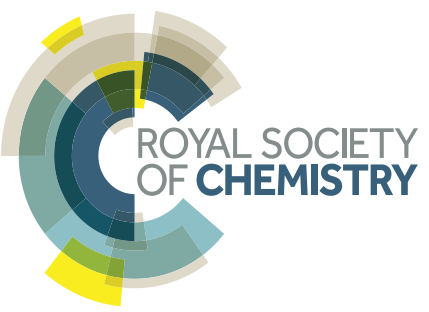




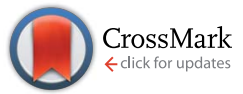

Cite this: Energy Environ. Sci., 2015, 8, 278

Received 23rd June 2014

Accepted 1st September 2014

DOI: 10.1039/c4ee01943a

www.rsc.org/ees

\section{Doping and electronic properties of GaAs grown by close-spaced vapor transport from powder sources for scalable III-V photovoltaics $\dagger$}

\author{
Andrew J. Ritenour, ${ }^{a}$ Jason W. Boucher, ${ }^{b}$ Robert DeLancey, ${ }^{b}$ Ann L. Greenaway, ${ }^{a}$ \\ Shaul Aloni ${ }^{\mathrm{C}}$ and Shannon W. Boettcher ${ }^{\star a}$
}

We report the use of a simple close-spaced vapor transport technique for the growth of high-quality epitaxial GaAs films using potentially inexpensive GaAs powders as precursors. The free carrier type and density $\left(10^{16}\right.$ to $10^{19} \mathrm{~cm}^{-3}$ ) of the films were adjusted by addition of Te or $\mathrm{Zn}$ powder to the GaAs source powder. We show using photoelectrochemical and electron beam-induced current analyses that the minority carrier diffusion lengths of the $\mathrm{n}$ - and $\mathrm{p}$-GaAs films reached $\sim 3 \mu \mathrm{m}$ and $\sim 8 \mu \mathrm{m}$, respectively. Hall mobilities approach those achieved for GaAs grown by metal-organic chemical vapor deposition, $1000-4200 \mathrm{~cm}^{2} V^{-1} \mathrm{~s}^{-1}$ for $\mathrm{n}-\mathrm{GaAs}$ and $50-240 \mathrm{~cm} \mathrm{~V}^{-1} \mathrm{~s}^{-1}$ for $\mathrm{p}-\mathrm{GaAs}$ depending on doping level. We conclude that the electronic quality of GaAs grown by close-spaced vapor transport is similar to that of GaAs made using conventional techniques and is thus sufficient for high-performance photovoltaic applications.

\section{Broader context}

The high balance-of-system costs of photovoltaic (PV) installations indicate that reductions in cell \$ per W costs alone are likely insufficient for PV electricity to reach grid parity unless energy conversion efficiency is also increased. Technologies which yield both high-efficiency cells (>25\%) and maintain low costs are needed. GaAs and related III-V semiconductors are used in the highest-efficiency single- and multi-junction photovoltaics, but the technology is too expensive for non-concentrated terrestrial applications. This is due in part to the difficulty of scaling the metal-organic chemical vapor deposition (MOCVD) process, which relies on expensive reactors and employs toxic and pyrophoric gas-phase precursors such as arsine and trimethyl gallium, respectively. We describe GaAs films made by an alternative close-spaced vapor transport (CSVT) technique which is carried out at atmospheric pressure and requires only bulk GaAs, water vapor, and a temperature gradient in order to deposit crystalline films with similar electronic properties to that of GaAs deposited by MOCVD. CSVT is similar to the vapor transport process used to deposit CdTe thin films and is thus a potentially scalable low-cost route to GaAs thin films.

\section{Introduction}

GaAs is interesting for photovoltaic (PV) applications due to its high carrier mobilities, large optical absorption coefficient $\alpha(\lambda)$, and direct band gap of $E_{\mathrm{g}}=1.42 \mathrm{eV}$. These properties lead to a theoretical maximum one-sun energy conversion efficiency of $\eta=33.5 \%$ (ref. 1-4) and demonstrated world-record of $\eta=28.8 \% .^{5}$ However, GaAs is not currently cost-competitive with Si or CdTe flat-panel PVs due in part to the high cost and relatively low throughput of conventional epitaxial growth techniques. ${ }^{6}$ Low-cost, high-throughput routes to GaAs films are needed.

${ }^{a}$ Department of Chemistry and Biochemistry, University of Oregon, Eugene, 97403, USA.E-mail: swb@uoregon.edu

${ }^{b}$ Department of Physics, University of Oregon, Eugene, 97403, USA

'The Molecular Foundry, Lawrence Berkeley National Laboratory, Berkeley, California 94720, USA

$\uparrow$ Electronic supplementary information (ESI) available. See DOI: 10.1039/c4ee01943a
Close-spaced vapor transport (CSVT) is a plausibly scalable technique for making epitaxial GaAs films. ${ }^{7-10}$ In CSVT, $\mathrm{H}_{2} \mathrm{O}$ vapor (typically $\sim 2000 \mathrm{ppm}$ in hydrogen or forming gas) is used to etch a solid GaAs source, generating vapor-phase reactants $\mathrm{As}_{2}$ and $\mathrm{Ga}_{2} \mathrm{O}$ in situ at atmospheric pressure (Fig. 1). ${ }^{11}$ The liberated $\mathrm{As}_{2}$ and $\mathrm{Ga}_{2} \mathrm{O}$ diffuse through a thermal gradient (typically $\Delta T=10-50{ }^{\circ} \mathrm{C}$ ) and re-deposit as GaAs on the cooler substrate suspended $<1 \mathrm{~mm}$ from the source by a spacer. The CSVT reactor can be relatively simple, inexpensive, and compact. CSVT is capable of high growth rate (up to $1 \mu \mathrm{m} \mathrm{min} \mathrm{m}^{-1}$

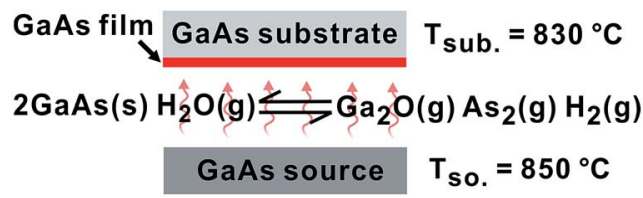

Fig. 1 Schematic of CSVT of GaAs with in situ generation of gas-phase precursors. 
demonstrated) and $>95 \%$ overall precursor transport/utilization efficiency from source to substrate. ${ }^{8}$ The supersaturation can be tuned at the substrate through control of the temperature gradient, facilitating selected-area epitaxy and epitaxial layer overgrowth (ELO) on Si substrates. ${ }^{12,13}$ These features could potentially enable growth of high-quality GaAs films with large grains $^{\mathbf{1 4}}$ on thermally/lattice-mismatched ${ }^{\mathbf{1 5 , 1 6}}$ or ceramic $^{\mathbf{1 7}}$ substrates. This is significant because in order to successfully utilize GaAs for terrestrial PV applications, in addition to developing an efficient growth technique, the substrate must either be inexpensive or reusable (e.g. through epitaxial lift-off processes). ${ }^{6}$

The use of $\mathrm{H}_{2} \mathrm{O}$ as a transport agent is an important advantage of CSVT over the conventional metal-organic chemical vapor deposition (MOCVD) process. Although MOCVD produces high-mobility GaAs films, it relies on the use of gasphase precursors such as arsine (acutely toxic) and trimethylgallium (pyrophoric). The use of these hazardous and expensive precursors contributes to the cost and complexity of MOCVD reactors and makes it less appealing for the growth of GaAs for terrestrial PV applications, which require both low cost and high throughput.

Liquid phase epitaxy (LPE) has also been used for GaAs epitaxy yielding high-mobility (equivalent to MOCVD films) GaAs and also high growth rates $\left(>1 \mu \mathrm{m} \mathrm{min}^{-1}\right)$, and thus is also appealing for PV applications. LPE is, however, a batch process with likely lower throughput to what is ultimately possible with vapor phase deposition. ${ }^{18}$ It is also difficult to control/ implement heteroepitaxy, especially on lower cost substrates such as Si.

Despite the merits of the CSVT technique, few CSVT GaAs devices have been reported. ${ }^{\mathbf{1 9 - 2 1}}$ We previously demonstrated CSVT n-GaAs films with minority carrier diffusion lengths $\left(L_{\mathrm{D}}\right)>$ $1 \mu \mathrm{m}$ (ref. 22) and overall $\eta=9.3 \%$ in a photoelectrochemical (PEC) cell, ${ }^{23}$ nearly equivalent to MOCVD n-GaAs photoanodes $(\eta=11 \%){ }^{24}$ These CSVT $\mathrm{n}$-GaAs films were grown from Sidoped GaAs sources and exhibited free electron concentrations of $0.5-2 \times 10^{17} \mathrm{~cm}^{-3}$. We have since determined that these are doped by $\mathrm{S}$ (which outgasses from the graphite heaters upon heating) rather than transport of Si dopants from the Si-doped GaAs source. The $\mathrm{S}$ doping is discussed further below (see Results and discussion). Controlling the dopant type and dopant density of CSVT GaAs films is a key step toward fabrication of solid-state PV devices. For PV applications, the films must also possess high carrier mobilities and $L_{\mathrm{D}}>\alpha(\lambda)^{-1}$.

Here we report n- and p-GaAs films with a range of free electron and free hole concentrations $\left(N_{\mathrm{D}}\right.$ and $N_{\mathrm{A}}$, respectively) grown using CSVT from potentially low-cost powder sources. Mixing powders could also be used to access related ternary III$\mathrm{V}$ materials such as $\operatorname{GaAs}_{x} \mathrm{P}_{1-x} \cdot{ }^{25} N_{\mathrm{D}}$ and $N_{\mathrm{A}}$ determined from impedance and Hall-effect measurements agree with dopant concentrations obtained from secondary ion mass spectrometry (SIMS), and demonstrate control over the dopant concentration from $\sim 10^{16} \mathrm{~cm}^{-3}$ to $\sim 10^{19} \mathrm{~cm}^{-3}$. The $L_{\mathrm{D}}$ was up to $\sim 3 \mu \mathrm{m}$ for $\mathrm{n}$-GaAs films and up to $\sim 8 \mu \mathrm{m}$ for $\mathrm{p}$-GaAs films, determined independently via analysis of the internal quantum efficiency $\Phi_{\text {int }}$ and electron beam induced current (EBIC). These $L_{\mathrm{D}}$ are long with respect to $\alpha(\lambda)^{-1}$ and consistent with the measured one-sun photocurrents in the PEC configuration $\left(>20 \mathrm{~mA} \mathrm{~cm}^{-2}\right.$ with no antireflective coating). Hall mobilities of CSVT n- and p-GaAs approach the ionized dopant scattering $\operatorname{limit}^{26}$ and are similar to what has been achieved using MOCVD. ${ }^{27}$

These results demonstrate that potentially inexpensive powdered GaAs can be used to deposit GaAs films suitable for high performance III-V based PV devices ${ }^{28}$ at high growth rate, with $\sim 95 \%$ precursor utilization, and at ambient pressures using a simple CSVT reactor.

\section{Experimental}

\section{GaAs thin film deposition}

The carrier gas for CSVT was $\mathrm{H}_{2}$ (Industrial Source, 99.999\%) with water concentration $\left[\mathrm{H}_{2} \mathrm{O}\right]=2000$ ppm. $\left[\mathrm{H}_{2} \mathrm{O}\right]$ was controlled by combining a stream of dry $\mathrm{H}_{2}$ with a stream of $\mathrm{H}_{2} \mathrm{O}$-saturated (dew point $10^{\circ} \mathrm{C}$ ) $\mathrm{H}_{2}$ using mass flow controllers (SEC 4400) and monitored with a Panametrics MM4 Hygrometer. PID controllers (Omega CN7800) were used to set the growth temperature to $850{ }^{\circ} \mathrm{C}$ and $830{ }^{\circ} \mathrm{C}$ for the source and substrate, respectively, as shown in Fig. 1. The temperature was monitored using type $\mathrm{K}$ thermocouples embedded in the resistive graphite heaters. A quartz ring $12 \mathrm{~mm}$ in diameter and $0.8 \mathrm{~mm}$ thick was used as a spacer between the source and substrate. The film thicknesses of all PEC samples were 5-11 $\mu \mathrm{m}$ (measurements obtained from a Zygo 7300 optical profilometer), ensuring all light was absorbed in the CSVT GaAs film rather than the substrate. The growth rate (which depends on

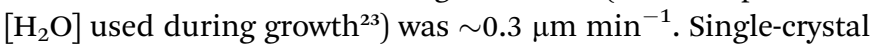
$\langle 100\rangle$-oriented GaAs wafers grown by the vertical gradient freeze technique $^{29}$ (AXT, Inc.) were used as substrates. The substrates were epi-ready as received and cleaned by blowing with $\mathrm{N}_{2}$. More details about the CSVT reactor are provided in previous publications. ${ }^{22,23}$

Powder GaAs sources were obtained by grinding undoped GaAs wafers (AXT) in an agate mortar and pestle and pressing at $140 \mathrm{MPa}$ in a $13 \mathrm{~mm}$ pellet die. The mortar and pestle were cleaned by submersing in aqua regia and rinsing with 18.2 $\mathrm{M} \Omega$ $\mathrm{cm}$ water. Zn powders were separately weighed and combined with the GaAs powder prior to pressing. Te-doped powders with $[\mathrm{Te}]<10^{19} \mathrm{~cm}^{-3}$ were made by grinding and pressing Te-doped wafers. A source pellet containing $[\mathrm{Te}]=10^{19} \mathrm{~cm}^{-3}$ was made by combining undoped GaAs and Te powder. Single-crystal wafers were also used as sources to provide a comparison to the powders. These were cut into $13 \times 13 \mathrm{~mm}$ squares and cleaned by blowing with $\mathrm{N}_{2}$.

\section{Photoelectrochemical measurements}

Electrodes were fabricated using standard procedures. ${ }^{23}$ Ohmic contacts were formed on the back of the substrates by thermal evaporation of $\mathrm{Au} / \mathrm{Zn} / \mathrm{Au}(20 \mathrm{~nm}, 30 \mathrm{~nm}, 50 \mathrm{~nm})$ for $\mathrm{p}-\mathrm{GaAs}$ or AuGe eutectic $(100 \mathrm{~nm})$ for $\mathrm{n}-\mathrm{GaAs}$. Contacts were annealed at $450{ }^{\circ} \mathrm{C}$ for $2 \mathrm{~min}$ in $95 \% \mathrm{~N}_{2} / 5 \% \mathrm{H}_{2} \cdot{ }^{30}$ Ohmic contacts were connected to $\mathrm{Sn}-\mathrm{Cu}$ wire with conductive $\mathrm{Ag}$ adhesive (Pelco 16040-30) and attached to $6 \mathrm{~mm}$ diameter glass tubes with 
non-conductive epoxy (Hysol 1C). A circular electrode active region $\left(\sim 0.05 \mathrm{~cm}^{2}\right)$ was defined using non-conductive black epoxy (Hysol 9460).

Rectifying contacts to n-GaAs for current-voltage $(J-E)$ measurements, impedance measurements, and spectral response measurements were obtained using an electrolyte consisting of $1 \mathrm{M} \mathrm{LiClO}_{4}$ (Alfa-Aesar, 99\%, anhydrous), $100 \mathrm{mM}$ ferrocene (Aldrich, 98\%, sublimed before use), and $0.5 \mathrm{mM}$ ferrocenium tetrafluoroborate (obtained by oxidizing ferrocene with benzoquinone in the presence of $\mathrm{HBF}_{4}$, recrystallizing in tetrahydrofuran, and drying under vacuum) in dry acetonitrile (Acros, 99.8\%, distilled and dried with freshly-activated $3 \AA$ molecular sieves)..$^{24,31}$ For spectral response measurements, the solution was diluted $1: 10$ with dry acetonitrile in order to reduce parasitic solution absorbance.

For one-sun $J-E$ measurements of p-GaAs, an aqueous solution of $1 \mathrm{M} \mathrm{HI}$ (Aldrich, 99.99\%) and $0.125 \mathrm{M} \mathrm{I}_{2}$ (Alfa-Aesar, $99.8+\%)$ was used. ${ }^{32,33}$ For spectral response and impedance of p-GaAs a non-aqueous electrolyte consisting of $0.1 \mathrm{M} \mathrm{NaI}$ (AlfaAesar, 99+\%, anhydrous), $0.0125 \mathrm{M} \mathrm{I}_{2}$ (Sigma-Aldrich 99.99\%, sublimed), and $0.1 \mathrm{M} \mathrm{LiClO}_{4}$ in dry acetonitrile was used (see Results and discussion below).

For all PEC measurements a potentiostat (Bio-Logic SP-200) in three-electrode configuration was used. The GaAs electrode potential $(E)$ was referenced to the potential of a Pt wire poised at the solution potential $\left(E_{\mathrm{sol}}\right)$ and a Pt mesh was used as the counter electrode. The three electrodes were held in a glass three-neck flask containing the appropriate electrolyte with the GaAs electrode $<1 \mathrm{~mm}$ from the bottom surface. Mass transport was aided by a magnetic stirrer. Illumination was provided by a solar simulator (Abet Technologies model 10500) for $J-E$ experiments. The light intensity incident on the front face of the glass cell was $100 \mathrm{~mW} \mathrm{~cm}{ }^{-2}$ as determined using a calibrated photodiode (OSI Optoelectronics UV-005). The photodiode was calibrating using an optical pyrometer (Thor Labs S310C).

\section{Spectral response}

Spectral response of the PEC cells was measured using a Bentham PVE300 system. The monochromatic light was chopped at $35 \mathrm{~Hz}$ and the nA-range signal was measured using a lock-in amplifier. The chopped signal was free of transients and the amplitude of the signal was independent of chopping frequency from $10-50 \mathrm{~Hz}^{34} \mathrm{~A}$ calibrated photodiode (Bentham 11677) was used to determine the incident light intensity so that the nA signal could be converted to external quantum efficiency $\left(\Phi_{\text {ext }}\right)$. The chopped photocurrent scaled linearly with the incident light intensity over five orders of magnitude (Fig. S6 †).

Reflectance, $R(\lambda)$, of the air|glass|acetonitrile|GaAs stack was measured using a spectrometer with an integrating sphere (Perkin Elmer Lambda 1050). ${ }^{34}$ This data was used to obtain $\Phi_{\text {int }}$ from $\Phi_{\text {ext }}=\Phi_{\text {int }}[1-R(\lambda)]$. Although films from wafer sources were not completely specular, all films possessed $R(\lambda)$ equivalent to a polished single-crystal GaAs wafer when measured in the integrating sphere. Therefore, although some of the films exhibited diffuse reflectance, the total $R(\lambda)$ was unchanged. All films grown from powder sources were specular.

\section{Impedance spectroscopy}

Impedance measurements were conducted with a potentiostat on GaAs electrodes in a dark box. The DC reverse bias was varied between $0-1 \mathrm{~V}$ in 10 steps for $\mathrm{n}-\mathrm{GaAs}$ and 0 to $-0.5 \mathrm{~V}$ in 10 steps for p-GaAs electrodes with a $10 \mathrm{mV}$ AC bias amplitude. The impedance data were fit to the typical equivalent circuit model to extract the junction capacitance $C$ (Fig. S4 $\dagger$ ). ${ }^{35}$ Mott-Schottky analysis was used to determine $N_{\mathrm{D}}$ or $N_{\mathrm{A}}$ and the barrier height $V_{\text {bi }}$ (Fig. S5†). . $^{31,36}$

$$
\frac{1}{C^{2}}=\frac{2\left(V_{\mathrm{bi}}-V_{\mathrm{app}}-k T / q\right)}{q \varepsilon N A^{2}}
$$

The depletion region thickness $W$ was obtained using: ${ }^{37}$

$$
W=\sqrt{\frac{2 \varepsilon}{q N}\left(V_{\mathrm{bi}}-V_{\mathrm{app}}-k T / q\right)}
$$

where $\varepsilon$ is the dielectric constant of GaAs, $q$ is the fundamental charge, $V_{\text {app }}$ is the applied bias, $k$ is the Boltzman constant, and $T$ is the temperature.

\section{Hall effect measurements}

GaAs films deposited on undoped semi-insulating (resistivity $\rho$ $\left.>10^{7} \Omega \mathrm{cm}\right)$ substrates were cut into squares $\left(0.49-0.64 \mathrm{~cm}^{2}\right)$ and ohmic contacts were applied to the corners. The carrier type, $\rho, N_{\mathrm{A}}$ or $N_{\mathrm{D}}$, and majority carrier mobility $\left(\mu_{\mathrm{e}}\right.$ or $\mu_{\mathrm{h}}$ for electron or hole mobility, respectively) were obtained using the Van der Pauw method. ${ }^{38}$ Hall effect measurements were conducted at $300 \mathrm{~K}$ on an Ecopia HMS-5000 Hall effect system.

\section{Time-of-flight secondary ion mass spectrometry}

Mass spectra were obtained from a Cameca ION-TOF mass spectrometer with $\mathrm{Cs}^{+}$sputter gun and $\mathrm{Bi}^{+}$analysis gun. Ion counts were converted to bulk impurity concentrations using relative sensitivity factors (RSF).

$$
[\mathrm{E}]=\mathrm{RSF}_{\mathrm{E}} \times \frac{I_{\mathrm{E}}}{I_{\mathrm{M}}}
$$

where $[\mathrm{E}]$ is the concentration of the element being analyzed in units of $\mathrm{cm}^{-3}, \mathrm{RSF}_{\mathrm{E}}$ is the relative sensitivity factor of the element in the GaAs matrix, $I_{\mathrm{E}}$ is the signal of the element of interest, and $I_{\mathrm{M}}$ is the signal of the GaAs matrix (Fig. S1 $\dagger$ ). Values for RSF were obtained from the literature. ${ }^{39}$ To confirm the validity of published RSF values with our experiment, we measured two ion implanted samples (one ${ }^{28} \mathrm{Si}$-implanted and one ${ }^{16} \mathrm{O}$-implanted) and compared the obtained RSF values to the literature values. In both cases the values agreed to within $\pm 10 \%$. We also used TOF-SIMS to measure the [Te] and [Si] of Te-doped and Si-doped GaAs wafers purchased from AXT. Measured [Te] and [Si] agreed with both the vendor specifications and with $N_{\mathrm{D}}$ determined from impedance analysis. One CSVT-deposited p-GaAs film was sent to QSPEC for [Zn] determination on a magnetic sector SIMS, which possesses higher sensitivity to Zn than TOF-SIMS. 


\section{Electron beam-induced current}

The EBIC measurements were conducted in a scanning electron microscope (FEI Quanta 200F). The electron beam was scanned toward the Schottky contact and the resulting current transients (Fig. S7 $\dagger$ ) were recorded from a Matelect ISM-6A induced signal monitor. $L_{\mathrm{D}}$ was extracted from the decays according to:

$$
I=q N_{\mathrm{C}} \mathrm{e}^{-x / L_{\mathrm{D}}}
$$

where the beam-induced current $I$ is proportional to the exponential term, $N_{\mathrm{C}}$ is the number of minority carriers generated by the excitation beam per second, and $x$ is the separation between the excitation beam target and the Schottky junction. ${ }^{\mathbf{4 0}}$ The Schottky contact pads were $50 \mu \mathrm{m}$ gold squares patterned using photolithographic lift-off with a negative photoresist (AZ 5214E).

Prior to EBIC experiments, surface passivation was needed to lower the surface recombination velocity (SRV). Surface passivation was accomplished by etching with $5 \mathrm{M} \mathrm{HCl}$, rinsing with 18.2 $\mathrm{M} \Omega \mathrm{cm}$ water, immersing in aqueous $1 \mathrm{M} \mathrm{Na}_{2} \mathrm{~S}^{41}$ rinsing with water and ethanol, and drying with $\mathrm{N}_{2}$. Samples were immediately pumped into the vacuum chamber and measured within $20 \mathrm{~min}$ after passivation. Results obtained without passivation varied as a function of accelerating voltage $\left(V_{\text {acc }}\right)$ and did not produce reliable values of $L_{\mathrm{D}}$ (see Results and discussion). The signal decays used to extract $L_{\mathrm{D}}$ fit eqn (4) over several orders of magnitude of current (Fig. S7 $\dagger$ ).

\section{Results and discussion}

\section{Growth and doping from powder and wafer sources}

In order to fabricate high-efficiency GaAs PV devices, it is important to be able to control both $N_{\mathrm{A}}$ and $N_{\mathrm{D}}$ while maintaining suitable electronic quality of the GaAs layers. Thus to demonstrate the utility of the CSVT technique, it is necessary to determine which dopants are transported by CSVT, how the source dopant concentration is related to the film dopant concentration, and how they affect the electronic quality of the layers.

We previously reported the growth of n-GaAs films by CSVT from Si-doped wafer sources and hypothesized that the films were Si-doped since the $N_{\mathrm{D}}$ of the films $\left(\sim 3 \times 10^{17} \mathrm{~cm}^{-3}\right)$ matched the $[\mathrm{Si}]$ in the source wafer. However, upon TOF-SIMS analysis we determined that the films were S-doped rather than Si-doped (Fig. S1†). The poor transport efficiency of Si is likely related to the low vapor pressure of $\mathrm{SiO}_{x}$, which forms at high temperatures in the presence of $\mathrm{H}_{2} \mathrm{O}$. Unintentional S-doping of GaAs layers grown by CSVT has also been reported in other studies. ${ }^{\mathbf{4 2 , 4 3}}$

The unintentional S impurity is undesirable because it is a compensating defect in p-GaAs films. We used TOF-SIMS to determine that the graphite heaters were a source of $S$ impurity (Fig. S2 $\dagger$ ). After fabricating purified heaters, the unintentional S-doping of the films decreased to $[\mathrm{S}] \leq 7 \times 10^{16} \mathrm{~cm}^{-3}$ as determined by TOF-SIMS analysis of the films. Although the [S] could likely be further reduced by using non-porous, higherpurity heater materials (e.g. more-expensive pyrolysis-derived graphite), for this study the [S] achieved was low enough to permit growth of p-GaAs films with $N_{\mathrm{A}} \geq 10^{17} \mathrm{~cm}^{-3}$, which is appropriate for use as a p-type absorbing layer in a PV device.

$\mathrm{Zn}$ is widely used as a p-type dopant in GaAs and has been shown to transport by CSVT. ${ }^{43}$ GaAs films grown using CSVT from $\mathrm{Zn}$-doped wafers possess $\sim 1 / 100^{\text {th }}$ the $N_{\mathrm{A}}$ of the source wafer. ${ }^{43}$ Commercial GaAs wafers were available with [Zn] $<2 \times$ $10^{19} \mathrm{~cm}^{-3}$, setting an upper limit of $N_{\mathrm{A}} \approx 2 \times 10^{17} \mathrm{~cm}^{-3}$ for CSVT films grown from commercially available sources (practically less, due to $\mathrm{S}$ compensation). This is problematic since some active $\mathrm{PV}$ device components (e.g. emitters and back surface fields) require $N_{\mathrm{A}}>2 \times 10^{17} \mathrm{~cm}^{-3}$. In order to grow p-GaAs films with higher $N_{\mathrm{A}}$, we mixed GaAs and $\mathrm{Zn}$ powders at the desired ratio and pressed the mixtures into pellets. These pellet sources yielded p-GaAs films with $N_{\mathrm{A}}$ up to $\sim 2 \times 10^{19}$ $\mathrm{cm}^{-3}$ (Table 1).

In order to control $N_{\mathrm{D}}$, we used Te-doped GaAs sources. It has been shown using impedance profiling that GaAs films grown using CSVT from Te-doped wafers possess $N_{\mathrm{D}}$ equivalent to the source wafers. ${ }^{43-45}$ We reproduced these results by using two nGaAs:Te sources with different [Te] to grow GaAs films on degenerately-doped GaAs:Si substrates and measuring $N_{\mathrm{D}}$ with impedance profiling. In order to confirm that the dopants were transported by CSVT and not diffused from the substrate, we also deposited n-GaAs:Te films on undoped, semi-insulating substrates. Hall effect measurements of these samples confirm the same relationship, $N_{\mathrm{D}} \approx$ source [Te]. We also show using TOF-SIMS that the films possess [Te] similar to the source's [Te] and contain no Si from the GaAs:Si substrate (Fig. 2). All of this data is summarized in Table 1.

\section{Photoelectrochemical $J-E$ analysis}

The PEC GaAs|electrolyte junction is a convenient tool which enables the study of material properties by analyzing photocurrent $v s$. potential $(J-E)$ response, junction impedance, and $\Phi_{\text {int }}$ without fabrication of solid state devices. ${ }^{23,24,31}$

Electrodes of CSVT n-GaAs films were immersed in a nonaqueous ferrocene/ferrocenium electrolyte $\left(\mathrm{Fc} / \mathrm{Fc}^{+}\right)$and their $J-E$ response was measured under $100 \mathrm{~mW} \mathrm{~cm}^{-2}$ of simulated AM1.5G irradiation. ${ }^{24}$ Commercial $\langle 100\rangle$-oriented single-crystal wafers were measured as controls. The CSVT samples produced open-circuit voltages $\left(V_{\mathrm{oc}}\right)$ up to $0.83 \mathrm{~V}$, equivalent to that attained by others using MOCVD n-GaAs. ${ }^{4,47}$ Short-circuit current density $\left(J_{\mathrm{sc}}\right)$ was $\sim 20 \mathrm{~mA} \mathrm{~cm} \mathrm{~cm}^{-2}$ for moderately-doped samples having $N_{\mathrm{D}}=10^{16}$ to $10^{17} \mathrm{~cm}^{-3}$. The performance of all samples exceed the respective bare substrates and similarlydoped GaAs control wafers (Fig. 3A). There were no significant differences between films grown from powder and wafer sources. Lower photocurrent was observed in highly-doped samples, which also exhibit lower $\mu_{\mathrm{h}}$ (and consequently $L_{\mathrm{D}}$ ) due to carrier scattering by ionized dopant atoms in the lattice (see Hall effect measurements below). ${ }^{48,49}$

Electrodes of CSVT p-GaAs films and control wafers were immersed in an aq. iodide/triiodide electrolyte $\left(\mathrm{I}^{-} / \mathrm{I}_{3}{ }^{-}\right)$and their $J-E$ response was measured under $100 \mathrm{~mW} \mathrm{~cm}^{-2}$ of simulated AM1.5G irradiation (Fig. 3B). ${ }^{32,33}$ The $V_{\text {oc }}$ was $0.15-0.20 \mathrm{~V} v s . E_{\mathrm{sol}}$, 
Table 1 Dopant densities (measured by impedance analysis and Hall effect) and impurity concentrations (determined by TOF-SIMS analysis) of several CSVT GaAs films deposited from powder and wafer sources

\begin{tabular}{|c|c|c|c|c|c|}
\hline $\begin{array}{l}\text { Powder or wafer } \\
\text { source }\end{array}$ & $\begin{array}{l}\text { Source dopant } \\
\text { species (E) }\end{array}$ & $\begin{array}{l}\text { Source }[\mathrm{E}]^{a} \\
\left(\mathrm{~cm}^{-3}\right)\end{array}$ & $\begin{array}{l}\text { Impedance analysis } N_{\mathrm{D}}-N_{\mathrm{A}}\left(\mathrm{cm}^{-3}\right) \\
\text { (average of three electrodes) }\end{array}$ & $\begin{array}{l}\text { Hall effect } N_{\mathrm{D}}- \\
N_{\mathrm{A}}\left(\mathrm{cm}^{-3}\right) \text { (one sample) }\end{array}$ & $\begin{array}{l}{[\mathrm{E}] \text { from SIMS }} \\
\left(\mathrm{cm}^{-3}\right) \text { (one sample) }\end{array}$ \\
\hline Wafer & $\mathrm{Te}$ & $2-4 \times 10^{18}$ & $3 \times 10^{18} \pm 1 \times 10^{18}$ & - & $2 \times 10^{18}$ \\
\hline Powder & $\mathrm{Te}$ & $2-4 \times 10^{18}$ & $4 \times 10^{18} \pm 1 \times 10^{18}$ & $3 \times 10^{18}$ & - \\
\hline Wafer & $\mathrm{Te}$ & $3-6 \times 10^{17}$ & $4 \times 10^{17} \pm 4 \times 10^{16}$ & $3 \times 10^{17}$ & $6 \times 10^{17}$ \\
\hline Powder & $\mathrm{Te}$ & $3-6 \times 10^{17}$ & $7 \times 10^{17} \pm 2 \times 10^{17}$ & $6 \times 10^{17}$ & - \\
\hline Powder & $\mathrm{Zn}$ & $5 \times 10^{21}$ & $-2 \times 10^{19} \pm 3 \times 10^{18}$ & $-4 \times 10^{19}$ & - \\
\hline Powder & $\mathrm{Zn}$ & $5 \times 10^{20}$ & $-4 \times 10^{18} \pm 7 \times 10^{17}$ & $-4 \times 10^{18}$ & - \\
\hline Wafer & $\mathrm{Zn}$ & $1-2 \times 10^{19}$ & $-2 \times 10^{17} \pm 1 \times 10^{16}$ & $-1 \times 10^{17}$ & $1 \times 10^{17}$ \\
\hline Powder & $\mathrm{S}^{b}$ & $-b$ & $1 \times 10^{17} \pm 4 \times 10^{16}$ & $2 \times 10^{16}$ & $3 \times 10^{16}$ \\
\hline Wafer & $S^{b}$ & $-^{b}$ & $7 \times 10^{16} \pm 3 \times 10^{16}$ & $8 \times 10^{16}$ & $7 \times 10^{16}$ \\
\hline
\end{tabular}

${ }^{a}$ For wafer sources the dopant density was provided by the manufacturer; for $\mathrm{Zn}$-doped powder sources the dopant density was calculated from the mass of the GaAs powder and the $\mathrm{Zn}$ powder used. ${ }^{b}$ The $\mathrm{S}$ dopant was not intentionally added.

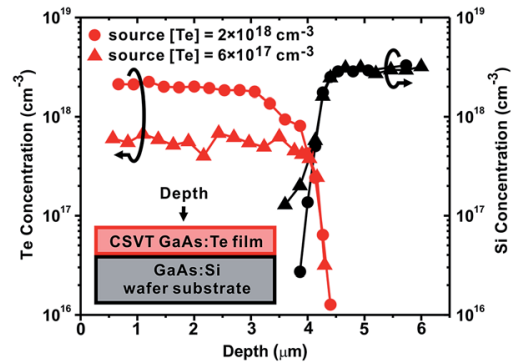

Fig. 2 SIMS depth profiles of two n-GaAs films grown from differently-doped GaAs:Te sources on GaAs:Si substrates. The Te depth profiles are shown in red and $\mathrm{Si}$ in black. Circles denote the film was grown from a source containing $[\mathrm{Te}]=2 \times 10^{18} \mathrm{~cm}^{-3}$, triangles denote the film was grown from a source with $[\mathrm{Te}]=6 \times 10^{17} \mathrm{~cm}^{-3}$.
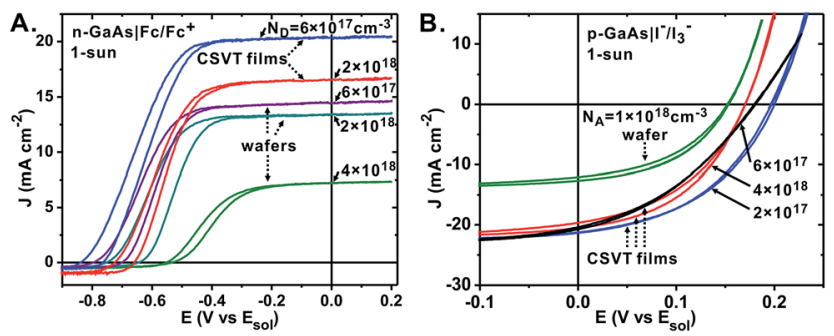

Fig. 3 Photoelectrochemical J-E curves of (A) n-GaAs and (B) p-GaAs CSVT films and control wafers. The curves are labeled with the corresponding sample's free carrier concentration in $\mathrm{cm}^{-3}$. The selected curves are representative of other electrodes obtained from the same samples and from other samples with similar free carrier concentrations.

lower than the n-GaAs samples due to surface pinning of the p-GaAs Fermi level near the valence band edge. ${ }^{32}$ The best samples exhibited $J_{\mathrm{sc}} \sim 20 \mathrm{~mA} \mathrm{~cm}^{-2}$, similar to the best $\mathrm{n}-\mathrm{GaAs}$ samples despite the higher parasitic light absorption of the $\mathrm{I}^{-} /$ $\mathrm{I}^{3-}$ electrolyte. All CSVT p-GaAs films (including those synthesized with $N_{\mathrm{A}}>10^{18} \mathrm{~cm}^{-3}$ ) exhibited higher photocurrent than the p-GaAs control wafer $\left(N_{\mathrm{A}}=1 \times 10^{18} \mathrm{~cm}^{-3}, J_{\mathrm{sc}}=12 \mathrm{~mA}\right.$ $\mathrm{cm}^{-2}$ ) indicating lower bulk recombination and a longer $L_{\mathrm{D}}$.

\section{Spectral response and $L_{\mathrm{D}}$ determination}

In order determine the $L_{\mathrm{D}}$ for each sample we measured the spectral response using the short-circuit PEC configuration $\left(E_{\mathrm{WE}}=0 \mathrm{~V} v s . E_{\mathrm{sol}}\right)$ under low-intensity chopped monochromatic light. For the n-GaAs films such measurements in the $\mathrm{Fc} / \mathrm{Fc}^{+}$ electrolyte are well-developed. Spectral response measurements of $\mathrm{p}$-GaAs in aqueous $\mathrm{I}^{-} / \mathrm{I}^{3-}$, however, were complicated by the solubility of GaAs in the acidic $\mathrm{I}^{-} / \mathrm{I}^{3-}$ electrolyte.

No etching of p-GaAs was observed in the aq. solution after hours of sustained operation as long as illumination was provided. However, a nA-range anodic current was observed in the aqueous solution when under dark or low-light conditions $\left(<1 \mu \mathrm{W} \mathrm{cm}^{-2}\right)$. This was problematic for spectral response, (which uses a nA-range chopped light source) and impedance analysis, which is conducted in the dark (Fig. S6†). We suspect the p-GaAs surface, while unstable in $\mathrm{H}_{2} \mathrm{O}$ especially at low $\mathrm{pH},{ }^{50}$ is cathodically stabilized by the photo-excited minority carrier electrons, causing it to act as a photo-gated battery.

Therefore we used a non-aqueous solution for the p-GaAs spectral response and impedance measurements. We used $\mathrm{NaI}$ to provide $\mathrm{I}^{-}$rather than HI. We also reduced the concentration of redox couple in order to decrease parasitic light absorbance. The low-concentration of redox couple in non-aqueous solution was sufficient to support the nA-range signal and exhibited no photo-gated current (Fig. S6†).

Trends for both $n$ and p-type GaAs in $J_{\mathrm{sc}}$ were mirrored by the spectral response curves (Fig. 4). Due to the wavelength dependence of $\alpha(\lambda)$, photons with energies near the band-gap $E_{\mathrm{g}}$ are absorbed further from the surface than those with higher energies. Thus $\Phi_{\text {int }}$ decays to zero at $E_{\mathrm{g}}$. This can be modeled using the Gärtner equation, which assumes no depletion region recombination and that the $L_{\mathrm{D}}$ governs bulk recombination:

$$
\Phi_{\text {int }}=\left(1-\frac{\mathrm{e}^{-\alpha(\lambda) W}}{1+\alpha(\lambda) L_{\mathrm{D}}}\right)
$$

where $W$ is the width of the semiconductor depletion region. ${ }^{51}$ This approach produces reliable estimates of $L_{\mathrm{D}}$ for $\mathrm{GaAs}^{52}$ and other semiconductors. ${ }^{53}$ Using this technique we measured three electrodes of each film. 

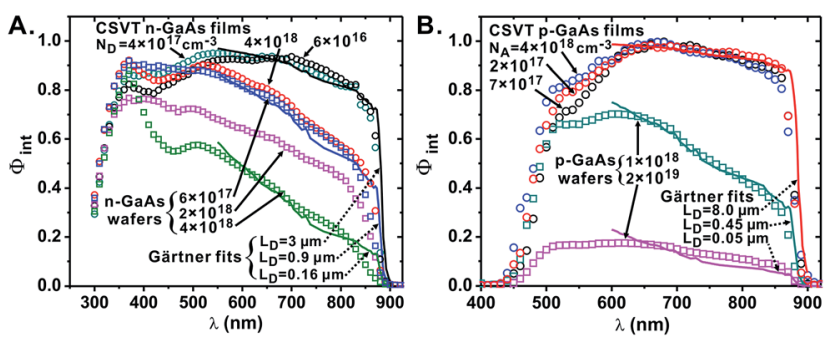

Fig. $4 \Phi_{\text {int }}$ measurements obtained using PEC on n-GaAs (A) and pGaAs samples (B). Experimental data is plotted as circles (CSVT GaAs films) or squares (commercial GaAs wafers) and calculated $L_{D}$ fits from eqn (5) are plotted as solid curves. The selected curves are representative of other electrodes obtained from the same samples and from other samples with similar free carrier concentrations.

One parameter fits to $\Phi_{\text {int }}$ from eqn (5) match the experimental data well (Fig. 4). Moderately-doped n-GaAs CSVT films have $L_{\mathrm{D}} \sim 2.9 \pm 0.2 \mu \mathrm{m}$, while CSVT $\mathrm{p}^{+}$-GaAs films possess $L_{\mathrm{D}}=$ $5.4 \pm 0.1 \mu \mathrm{m}$ and moderately-doped p-GaAs films possess $L_{\mathrm{D}}=$ $7.4 \pm 0.4 \mu \mathrm{m}$. The $L_{\mathrm{D}}$ is higher in p-GaAs because $\mu_{\mathrm{e}}$ is higher than $\mu_{\mathrm{h}}$, which in turn is due to the curvature of the conduction and valence bands. ${ }^{54}$ For all CSVT samples, the measured $L_{\mathrm{D}}$ was significantly higher than that of the control GaAs wafers $\left(L_{\mathrm{D}}=0.42 \mu \mathrm{m}\right.$ for $\mathrm{n}$-GaAs:Te, $0.16 \mu \mathrm{m}$ for $\mathrm{n}^{+}$-GaAs:Si, $0.45 \mu \mathrm{m}$ for p-GaAs:Zn, and $0.05 \mu \mathrm{m}$ for $\mathrm{p}^{+}$-GaAs:Zn) and consistent with one-sun $J_{\text {sc }}$ measurements (see above).

\section{Electron beam-induced current}

Because GaAs has a direct bandgap and large $\alpha(\lambda),{ }^{55}$ when $L_{\mathrm{D}}$ exceeds several $\mu \mathrm{m}$, the fraction of photons absorbed deeper than $L_{\mathrm{D}}+W$ is small, making determination of $L_{\mathrm{D}}$ by the Gärtner model less precise.

In EBIC analysis, the proximity of the excitation source to the charge separating junction is controlled by rastering an electron beam toward a Schottky contact (Fig. 5A), and is thus independent of $\alpha(\lambda)$. The beam-induced current is measured as a function of the distance between the junction and the excitation source, and $L_{\mathrm{D}}$ is determined by fitting the current decay according to eqn (4).
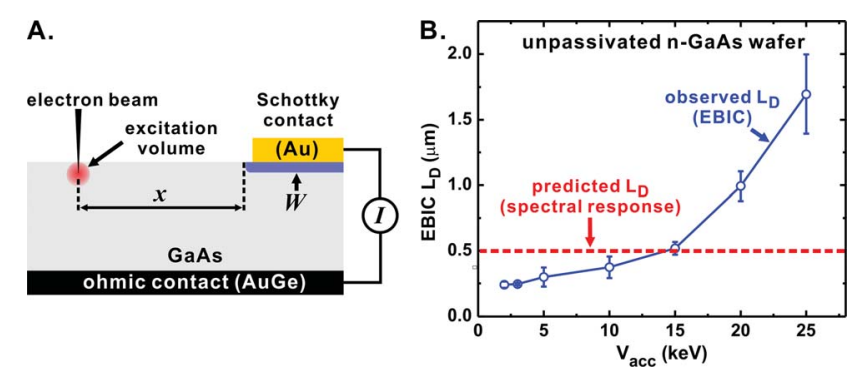

Fig. 5 (A) Schematic of the EBIC experiment. $X$ is the separation between the excitation source and the rectifying contact, $I$ is the induced current, and $W$ the depletion region. (B) The observed EBIC decay constant as a function of $V_{\text {acc }}$. The red dashed line indicates $L_{D}$ predicted by spectral response using the Gärtner method. The sample was an unpassivated $\mathrm{n}-\mathrm{GaAs}$ :Te wafer with $N_{\mathrm{D}}=6 \times 10^{16} \mathrm{~cm}^{-3}$.
After fabricating rectifying Au|n-GaAs junctions and measuring the EBIC response of the junctions, we observed that the response was a function of $V_{\text {acc }}$ (Fig. 5B), complicating accurate extraction of $L_{\mathrm{D}}$.

In an ideal EBIC experiment, the excitation volume (which is proportional to $V_{\text {acc }}$ ) is small with respect to $L_{\mathrm{D}}$ and the EBIC decay is dominated by bulk recombination with surface recombination playing a negligible role. These assumptions are invalid for unpassivated GaAs, which has a high SRV and a short $L_{\mathrm{D}}$ relative to indirect absorbers like Si. Thus at low $V_{\text {acc }}$ the EBIC decay is dominated by surface recombination yielding erroneously low $L_{\mathrm{D}}$, while at high $V_{\text {acc }}$ the excitation volume overlaps with the depletion region, yielding erroneously high $L_{\mathrm{D}}$. We note that in the experiments with unpassivated GaAs, the $L_{\mathrm{D}}$ obtained from EBIC analysis coincidentally agrees with spectral response when $V_{\text {acc }}=10-15 \mathrm{keV}$ is used, which matches the $V_{\text {acc }}$ used in other studies. ${ }^{52}$

In order to obtain EBIC data which is accurately modeled by eqn (4), we used $\mathrm{Na}_{2} \mathrm{~S}$ to passivate the GaAs surface thereby lowering the SRV. ${ }^{56}$ We also used a low $V_{\text {acc }} \leq 5 \mathrm{keV}$ in order to maintain a small interaction volume. After passivation, both spectral response and EBIC yielded similar values of $L_{\mathrm{D}}$ for $V_{\text {acc }} \leq 5 \mathrm{keV}$ (Fig. 6A). Comparing the two techniques we observe more dispersion in the EBIC results (Fig. 6B), but similar overall trends. We suspect the EBIC and spectral response results differ because spectral response averages the current over a relatively large region (generally $0.05 \mathrm{~cm}^{2}$ ), while EBIC measures the current decay of a line-scan and is therefore more sensitive to local recombination-inducing surface/bulk defects. Nonetheless, the direct measurement of $L_{\mathrm{D}}$ by EBIC using $\mathrm{Na}_{2} \mathrm{~S}$ passivation confirms the long $L_{\mathrm{D}}$ obtained from fitting PEC spectral response curves.

\section{Hall mobility and dopant density}

Films grown from a variety of $\mathrm{p}$ - and n-GaAs sources were deposited on undoped semi-insulating GaAs for Hall-effect measurements. The data indicates that n-GaAs and p-GaAs films deposited using CSVT (from both powder and wafer sources) have similar $\mu_{\mathrm{e}}$ and $\mu_{\mathrm{h}}$ to films deposited using MOCVD (Fig. 7). ${ }^{27,57}$

As $N_{\mathrm{D}}$ and $N_{\mathrm{A}}$ are increased, $\mu_{\mathrm{e}}$ and $\mu_{\mathrm{h}}$ decrease due to increased scattering from the ionized dopant atoms in the
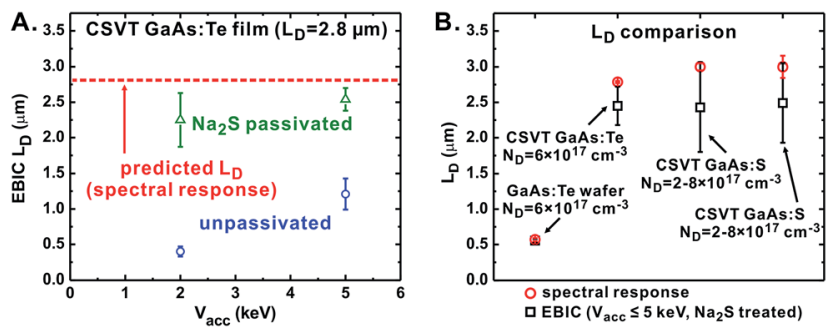

Fig. 6 (A) $L_{D}$ measured by EBIC on the same CSVT GaAs film before and after passivation by $\mathrm{Na}_{2} \mathrm{~S}$. The dashed line indicates the $L_{D}$ predicted by spectral response. (B) Comparison of $L_{D}$ obtained by spectral response and $\mathrm{EBIC}$ techniques. 

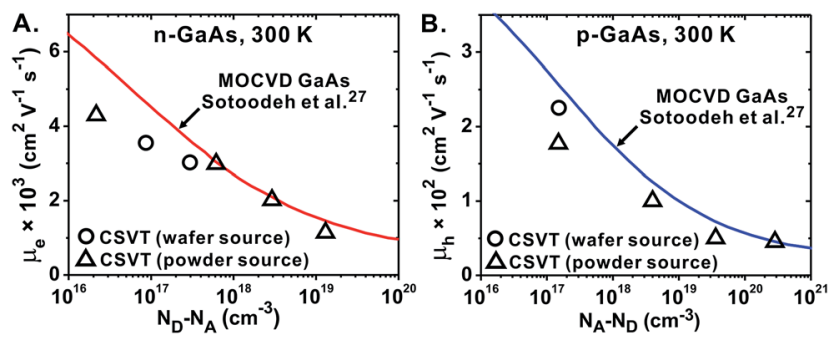

Fig. 7 Hall mobilities of $n-$ and $p$-GaAs films as a function of $N_{D}$ and $N_{\text {A. }}$ Solid curves represent the Hall mobility of high-quality epitaxiallygrown MOCVD GaAs. ${ }^{27}$

lattice (Fig. 7) ${ }^{26,48,49}$ The measured $\mu_{\mathrm{e}}$ and $\mu_{\mathrm{h}}$ of CSVT GaAs films deviate from the MOCVD values more for lightly-doped samples than for highly-doped samples. This is likely because at lower $N_{\mathrm{D}} / N_{\mathrm{A}}$, the influence of trace compensating impurities and crystal defects becomes important relative to the dopant atom scattering. Overall, these results indicate that CSVT from GaAs powder sources is competitive with MOCVD in terms of the achievable $\mu_{\mathrm{e}}$ and $\mu_{\mathrm{h}}$ for a wide range of $N_{\mathrm{D}} / N_{\mathrm{A}}$.

No significant differences were observed between films deposited from powder and wafer sources. This result is expected because the growth takes place at the interface between substrate and the gas phase. Thus the source's crystalline quality should not affect the CSVT process as long as it does not affect the ability of the surface to be etched by $\mathrm{H}_{2} \mathrm{O}$ to produce vapor phase $\mathrm{As}_{2}$ and $\mathrm{Ga}_{2} \mathrm{O}$. This implies that there is no need for crystalline powder sources, and lower quality powders could potentially be used as sources, for example those made by reaction of Ga and As at low temperatures. ${ }^{58}$

\section{Conclusions}

Epitaxial films of GaAs possessing a wide range of $N_{\mathrm{D}}$ and $N_{\mathrm{A}}$ were deposited via $\mathrm{H}_{2} \mathrm{O}$-mediated CSVT with growth rate $>0.3$ $\mu \mathrm{m} \min ^{-1}$ at ambient pressure from powdered GaAs precursors. $N_{\mathrm{D}}$ and $N_{\mathrm{A}}$ were measured and confirmed using complementary analyses which gave equivalent results (impedance spectroscopy, Hall effect measurements, and TOF-SIMS). This is an important step toward the fabrication of more complex device architectures such as $\mathrm{p}-\mathrm{n}$ junctions.

The room temperature mobilities of CSVT GaAs films were similar to those produced in the literature using MOCVD, despite the relatively high growth rates and use of $\mathrm{H}_{2} \mathrm{O}$ vapor as a transport agent. Due to the high $\alpha(\lambda)$ of GaAs, ${ }^{55}$ the $L_{\mathrm{D}}(2-3 \mu \mathrm{m}$ for n-GaAs and 5-8 $\mu \mathrm{m}$ for p-GaAs) was sufficient to yield $J_{\mathrm{sc}} \approx$ $20 \mathrm{~mA} \mathrm{~cm}^{-2}$ which is near the one-sun limit $\left(22.5 \mathrm{~mA} \mathrm{~cm}^{-2}\right)$ for specular GaAs with no anti-reflective coating in acetonitrile. For this $\mu_{\mathrm{e}}$ and $L_{\mathrm{D}}$ we estimate an electron lifetime of $\tau_{\mathrm{e}} \sim 7 \mathrm{~ns}$ for the $N_{\mathrm{A}}=1-2 \times 10^{17} \mathrm{~cm}^{-3} \mathrm{p}$-GaAs films. According to previously published simulations ${ }^{28}$ using this lifetime, $\eta \approx 25 \%$ singlejunction photovoltaics could be fabricated if other deviceengineering challenges (such as how to create rectifying solidstate junctions and passivate the surfaces) can be solved. Initial efforts to produce GaAs p-n junction PV solar cells using CSVT have produced promising results with $V_{\text {oc }}>0.9 \mathrm{~V}$ and $\Phi_{\text {int }}>0.9$, but they are beyond the scope of this study. ${ }^{59}$

In addition to the high capital cost associated with MOCVD production of GaAs, lattice-matched substrates are also expensive relative to Si wafers. Tandem architectures which utilize the larger indirect band-gap and smaller lattice constant (closer to Si) of $\mathrm{GaAs}_{x} \mathrm{P}_{1-x}$ could yield $\eta>35 \%$ devices on $\mathrm{Si}$ substrates if challenges associated with the thermal and lattice mismatch can be addressed. ${ }^{60}$ Growth techniques such as selective area epitaxy ${ }^{12}$ and the synthesis and use of engineered strain relaxation areas ${ }^{61}$ may be required for such efforts.

\section{Acknowledgements}

This work was supported by the Department of Energy (DOE) SunShot Initiative BRIDGE program (DE-EE0005957). AR acknowledges support from the Julie and Rocky Dixon Graduate Innovation Fellowship. AG acknowledges support from a NSF Graduate Research Fellowship (DGE-0829517). SB acknowledges support from Research Corporation for Science Advancement as a Cottrell Scholar. We acknowledge support from the user program at the Molecular Foundry, Lawrence Berkeley National Laboratory, with funding from the U.S. DOE Office of Science, Office of Basic Energy Sciences under Contract DE-AC02-05CH1123. The CAMCOR shared instrument facilities are supported by grants from the W.M Keck Foundation, the M.J. Murdock Charitable Trust, ONAMI, and NSF. The SUNRISE Photovoltaic Laboratory is supported by Oregon Built Environment and Sustainable Technologies. Assistance from Fuding Lin, Adam Smith, Stephen Golledge, Fred Stevie, Kris Johnson, Jeff Garmin, John Boosinger, Cliff Dax, Alex Negrete, Richard Cramer, Solomon Levinrad, Athavan Nadarajah, Lena Trotochaud, Matthew Kast, Sangeun Chun, Michaela Burke, T.J. Mills, Adam Batchellor and David Senkovich is acknowledged.

\section{Notes and references}

1 O. D. Miller, E. Yablonovitch and S. R. Kurtz, IEEE J. Photovolt., 2012, 2, 303-311.

2 W. Xufeng, M. R. Khan, J. L. Gray, M. A. Alam and M. S. Lundstrom, IEEE J. Photovolt., 2013, 3, 737-744.

3 L. S. Mattos, S. R. Scully, M. Syfu, E. Olson, Y. Linlin, C. Ling, B. M. Kayes and H. Gang, Proc. 38th IEEE Photovolt. Spec. Conf., 2012, pp. 3187-3190.

4 M. A. Green, K. Emery, Y. Hishikawa, W. Warta and E. D. Dunlop, Prog. Photovoltaics, 2014, 22, 1-9.

5 E. Yablonovitch, O. D. Miller and S. R. Kurtz, Proc. 38th IEEE Photovolt. Spec. Conf., 2012, pp. 1556-1559.

6 M. Bosi and C. Pelosi, Prog. Photovoltaics, 2007, 15, 51-68.

7 G. Perrier, R. Philippe and J. P. Dodelet, J. Mater. Res., 1988, 3, 1031-1042.

8 F. H. Nicoll, J. Electrochem. Soc., 1963, 110, 1165-1167.

9 E. Sirtl, J. Phys. Chem. Solids, 1963, 24, 1285-1289.

10 P. Robinson, RCA Rev., 1963, 24, 574-584.

11 M. Hammadi, J. C. Bourgoin and H. Samic, J. Mater. Sci.: Mater. Electron., 1999, 10, 399-402. 
12 M. G. Mauk, B. W. Feyock and J. E. Cotter, J. Cryst. Growth, 2001, 225, 528-533.

13 M. G. Mauk, A. N. Tata and B. W. Feyock, J. Cryst. Growth, 2001, 225, 359-365.

14 S. R. Kurtz and R. McConnell, AIP Conf. Proc., 1997, 404, 191205.

15 R. Venkatasubramanian, B. O'Quinn and E. Siivola, AIP Conf. Proc., 1997, 394, 259-270.

16 S. M. Vernon, S. P. Tobin, V. E. Haven, L. M. Geoffroy and M. M. Sanfacon, Proc. 22nd IEEE Photovolt. Spec. Conf., 1991, vol. 351, pp. 353-357.

17 M. G. Mauk, J. R. Balliet and B. W. Feyock, J. Cryst. Growth, 2003, 250, 50-56.

18 M. G. Astles, Liquid-Phase Epitaxial Growth of III-V Compound Semiconductor Materials and their Device Applications, WileyVCH Verlag GmbH, 1990.

19 M. G. Mauk, B. W. Feyock, R. B. Hall, K. D. Cavanaugh and J. E. Cotter, Proc. 26th IEEE Photovolt. Spec. Conf., 1997, pp. 511-514.

20 F. Silva-Andrade, A. Ilinskii, F. Chavez, T. Prutskij, F. Tenorio, Y. Bravo-Garcia and R. Pena-Sierra, Mod. Phys. Lett. B, 2001, 15, 752-755.

21 P. A. Hoss, L. Murray and J. Rivera, J. Electrochem. Soc., 1968, 115, 553-556.

22 A. J. Ritenour and S. W. Boettcher, Proc. 38th IEEE Photovolt. Spec. Conf., 2012, pp. 913-917.

23 A. J. Ritenour, R. C. Cramer, S. Levinrad and S. W. Boettcher, ACS Appl. Mater. Interfaces, 2012, 4, 69-73.

24 C. M. Gronet and N. S. Lewis, Appl. Phys. Lett., 1983, 43, 115117.

25 R. Purohit, J. Mater. Sci., 1968, 3, 330-332.

26 W. Walukiewicz, L. Lagowski, L. Jastrzebski, M. Lichtensteiger and H. C. Gatos, J. Appl. Phys., 1979, 50, 899-908.

27 M. Sotoodeh, A. Khalid and A. Rezazadeh, J. Appl. Phys., 2000, 87, 2890-2900.

28 J. W. Boucher, A. J. Ritenour and S. W. Boettcher, Proc. SPIE, 2013, 87250Z.

29 Crystal Growth Technology, ed. H. J. Scheel and P. Capper, Wiley-VCH, 2008.

30 V. Rideout, Solid-State Electron., 1975, 18, 541-550.

31 L. G. Casagrande, A. Juang and N. S. Lewis, J. Phys. Chem. B, 2000, 104, 5436-5447.

32 F. R. F. Fan and A. J. Bard, J. Am. Chem. Soc., 1980, 102, 36773683.

33 P. A. Kohl and A. J. Bard, J. Electrochem. Soc., 1979, 126, 5967.

34 A. J. Ritenour, S. Levinrad, C. Bradley, R. C. Cramer and S. W. Boettcher, ACS Nano, 2013, 6840-6849.

35 K. E. Pomykal, A. M. Fajardo and N. S. Lewis, J. Phys. Chem., 1995, 99, 8302-8310.

36 K. Gelderman, L. Lee and S. Donne, J. Chem. Educ., 2007, 84, 685.
37 S. M. Sze, Physics of Semiconductor Devices, John Wiley \& Sons, New York, 1981.

38 L. Van der Pauw, Philips Tech. Rev., 1958, 20, 220-224.

39 R. G. Wilson, F. A. Stevie and C. W. Magee, Secondary ion mass spectrometry: a practical handbook for depth profiling and bulk impurity analysis, Wiley New York, 1989.

40 B. Akamatsu, J. Henoc and P. Henoc, J. Appl. Phys., 1981, 52, 7245-7250.

41 B. J. Skromme, C. J. Sandroff, E. Yablonovitch and T. Gmitter, Appl. Phys. Lett., 1987, 51, 2022-2024.

42 J. Bourgoin, D. Stievenard, D. Deresmes and J. Mimila Arroyo, J. Appl. Phys., 1991, 69, 284-290.

43 C. Le Bel, D. Cossement, J. P. Dodelet, R. Leonelli, Y. DePuydt and P. Bertrand, J. Appl. Phys., 1993, 73, 12881296.

44 E. Koskiahde, D. Cossement, R. Paynter, J. P. Dodelet, A. Jean and B. A. Lombos, Can. J. Phys., 1989, 67, 251-258.

45 G. Gottlieb, J. Electrochem. Soc., 1965, 112, 192-196.

46 D. Cossement, J. P. Dodelet, T. Bretagnon, A. Jean and B. A. Lombos, J. Electrochem. Soc., 1991, 138, 830-834.

47 L. G. Casagrande, B. J. Tufts and N. S. Lewis, J. Phys. Chem., 1991, 95, 1373-1380.

48 D. L. Rode and S. Knight, Phys. Rev. B: Solid State, 1971, 3, 2534-2541.

49 E. F. Schubert, Doping in III-V semiconductors, Cambridge Univ Press, 1993.

50 G. K. Schweitzer and L. L. Pesterfield, The Aqueous Chemistry of the Elements, Oxfort University Press, 2010.

51 W. W. Gärtner, Phys. Rev., 1959, 116, 84.

52 B. J. Tufts, I. L. Abrahams, L. G. Casagrande and N. S. Lewis, J. Phys. Chem., 1989, 93, 3260-3269.

53 C. Xiang, G. M. Kimball, R. L. Grimm, B. S. Brunschwig, H. A. Atwater and N. S. Lewis, Energy Environ. Sci., 2011, 4, 1311-1318.

54 S. Adachi, Physical Properties of III-V Semiconductor Compounds, John Wiley \& Sons, 1st edn, 1992.

55 Handbook of Optical Constants of Solids, ed. E. D. Palik, Academic Press, Inc., 1985.

56 S. R. Lunt, G. N. Ryba, P. G. Santangelo and N. S. Lewis, J. Appl. Phys., 1991, 70, 7449-7467.

57 M. R. Brozel and G. Stillman, Properties of gallium arsenide, IET, 1996.

58 E. Fahrenkrug, J. Gu and S. Maldonado, J. Am. Chem. Soc., 2012, 135, 330-339.

59 J. W. Boucher, A. J. Ritenour, A. L. Greenaway, S. Aloni and S. W. Boettcher, Proc. 40th IEEE Photovolt. Spec. Conf., 2014.

60 J. Faucher, A. Gerger, S. Tomasulo, C. Ebert, A. Lochtefeld, A. Barnett and M. Lee, Appl. Phys. Lett., 2013, 103, 191901.

61 K. J. Schmieder, A. Gerger, M. Diaz, Z. Pulwin, C. Ebert, A. Lochtefeld, R. Opila and A. Barnett, Proc. 38th IEEE Photovolt. Spec. Conf., 2012, pp. 968-973. 